\title{
Power Minimization Under Constant Throughput Constraint in Wireless Networks with Beamforming
}

\author{
Zhu Han and K.J. Ray Liu, \\ Electrical and Computer Engineer Department, University of Maryland, College Park.
}

\begin{abstract}
In multi-access wireless communication systems, power control and adaptive modulation are two important means to increase spectral efficiency, combat with time varying fading environment, and reduce co-channel interference. In this paper, the overall transmitted power is minimized by using adaptive MQAM modulation. Each link can select a range of different MQAM modulation according to its current channel condition. Each link's time average throughput is a constant. The overall network throughput is a constant to ensure the network performance. The scheme can be interpreted as "water filling" each link's throughput in time domain and allocating overall throughput to different links at each time. From the simulation results, our schemes reduce up to $80 \%$ overall transmitted power at $B E R=10^{-3}, 66 \%$ overall transmitted power at $B E R=10^{-6}$, and increase average spectral efficiency by about $1.2 \mathrm{bit} / \mathrm{s} / \mathrm{Hz}$.
\end{abstract}

Keywords: Power Control, Adaptive Modulation, Beamforming, Co-channel Interference, Spectral Efficiency.

\section{INTRODUCTION}

The available wireless communication resources such as power and spectrum are extremely limited, while the demand for the services is growing rapidly. So how to optimally use the radio resources and increase the network throughput is therefore of primary concern in the design of future wireless communication systems. Two of the important detrimental effects to decrease network throughput are the time varying nature of the channel and co-channel interferences. Due to the time varying channel, the Signalto-Interference-Noise-Ratio (SINR) at the output of the receiver can fluctuate in the order of tens of dBs. Because the co-channel interferences reduce frequency-reuse distance, the network throughput for the areas is significantly decreased. One approach to combat these detrimental effects is to adapt the transmission power and modulation level based on the channel conditions. In power control, the transmitting powers are constantly adjusted. Such process improves the quality of weak links. But at the same time, it increases the co-channel interferences during the deep fading. In adaptive modulation, the system assigns the modulation levels with different spectral efficiency to different links according to their channel conditions. There are tradeoff and practical constraints to allocate these resources. So how to optimally manage these resources becomes a hot topic in nowadays wireless research.

In traditional power control[1], [2], each link's transmitting power is selected so that its SINR is equal to or larger than a fixed and predefined targeted SINR threshold required to maintain the link quality, while minimizing the overall transmitting power of all the links. However a link with bad channel response requires too much transmitting power and therefore causes unnecessary co-channel interference to other links. This is a major issue that we will address here. We concentrate on uplink situation. We introduce joint adaptive power and modulation allocation scheme using M-QAM modulation with beamforming. The goal of this paper is to minimize overall transmitting power. Instead of having fixed and predefined throughput, each user can select a range of modulation levels and throughputs according to its channel fading condition. In [3] and [4], it has been shown that the network throughput can be greatly improved by using adaptive modulation. However in their papers, the users with best channel conditions occupy most of transmission radio resources, which is very unfair in real communication system. In this paper, the time average throughput of each user is maintained as a constant to ensure the fairness. The overall network throughput is kept as a constant at each time to maintain the network performance. The scheme can be interpreted as "water filling" each user's throughput in time domain and allocating network throughput to different links at each time. A variable power and variable rate communication system is constructed. From the simulation results, our scheme reduces about $80 \%$ of overall transmitting power and increases average spectral efficiency by about $1.2 \mathrm{bit} / \mathrm{s} / \mathrm{Hz}$.

The organization of this paper is as follows: In Section II, we present system model. In section III, the optimization problem is formulated. The adaptive power and modulation management is developed. A variable power and variable rate communication system is constructed. In Section IV, we evaluate the performance by using simulation study. In Section V, we have the conclusion.

\section{System Models}

Consider K co-channel links existing in distinct cell in wireless networks. Each link consists of a mobile and its assigned base station. We assume coherent detection is possible so that it is sufficient to model this multiuser system by an equivalent base band model. Antenna arrays with $\mathrm{P}$ elements are used only at base station. Each link is affected by the multipath slow Rayleigh fading. The maximum multipath number is L. The propagation delay is far less than one symbol period. For uplink case, the output signal at the $i^{t h}$ base station antenna array is given by:

$$
\mathbf{x}_{i}(t)=\sum_{k=1}^{K} \sum_{l=1}^{L} \sqrt{\rho_{k i} G_{k i} \alpha_{k i}^{l} P_{k}} \mathbf{a}_{k i}\left(\theta_{l}\right) \cdot g_{k}(t) s_{k}(t)+\mathbf{n}_{i}(t)
$$




\section{MQAM Throughput vs. SINR}

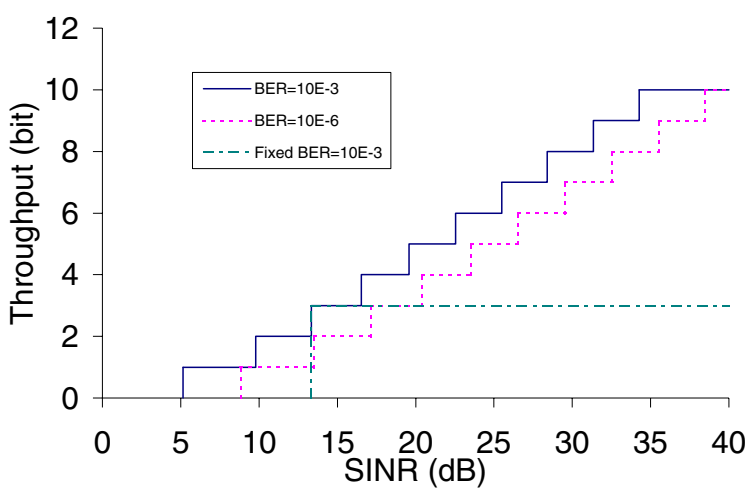

Fig. 1. Required SINR of MQAM for Desired BER

where $\alpha_{k i}^{l}$ is fading loss, $P_{k}$ is transmitted power, $\mathbf{a}_{k i}\left(\theta_{l}\right)$ is the $i^{t h}$ base station array response vector to the signal from $k^{t h}$ mobile at direction $\theta_{l}, g_{k}(t)$ is shaping function, $s_{k}(t)$ is message symbol, $\mathbf{n}_{i}(t)$ is thermal noise vector, $\rho_{k i}$ is $\log$ normal shadow fading and $G_{k i}$ is path loss. We assume slow fading and define the impulse response from the $k^{t h}$ mobile to the $p^{t h}$ element of the $i^{t h}$ base station as: $h_{k i}^{p}=\sum_{l=1}^{L} \sqrt{\alpha_{k i}^{l}} a_{k i}^{p}\left(\theta_{l}\right) r_{k i}^{p l}$, where $r_{k i}^{p l}$ includes the effect of the transmitter, receiver filter, and shaping function $g_{k}(t)$. The vector form is $\mathbf{h}_{k i}=\left[h_{k i}^{1}, \ldots, h_{m i}^{P}\right]^{T}$. We assume the transmitted signals from different sources are uncorrelated and zero mean, and the additive noise is spatially and temporally white. Let $\mathbf{w}_{i}$ be the beamforming weight vector. Without loss of generosity, let $\left\|\mathbf{w}_{i}^{H} \mathbf{h}_{i i}\right\|^{2}=1$. The $i^{\text {th }}$ link's Signal-to-Interference-Noise-Ratio (SINR) at the beamformer output is:

$$
\Gamma_{i}=\frac{P_{i} \rho_{i i} G_{i i}}{\sum_{k \neq i} P_{k} \rho_{k i} G_{k i}\left\|\mathbf{w}_{i}^{H} \mathbf{h}_{k i}\right\|^{2}+\mathbf{w}_{i}^{H} N_{i} \mathbf{w}_{i}}
$$

It has been shown that Bit Error Rate (BER) of square MQAM with Gray bit mapping as a function of received SINR $\Gamma$ and constellation size $M$ is approximately given by Equ. 3. We can calculate the required SINR for specific MQAM and require BER, then power control is applied so that each link has the required SINR.

$B E R_{M Q A M}(\Gamma) \approx \frac{2}{\log _{2} M}\left(1-\frac{1}{M}\right) \operatorname{erfc}\left(\sqrt{1.5 \frac{\Gamma}{M-1}}\right)$

\section{Power Minimization with Adaptive MODUlation}

\section{A. Problem Definition}

We assume the links can apply different modulation levels, according to their channel conditions. The overall network throughput is a constant to maintain the network performance and BER is ensured for each link. At each time, the links with bad channel conditions sacrifice their throughputs, i.e. they only need smaller constellation size and lower SINR thresholds; On the other hand, the links with good channel conditions get larger throughputs and higher SINR thresholds. For each link, the time average throughput is a constant to ensure the fairness. The scheme can be described as "water filling" each user's throughput in time domain and allocating overall network throughput to different links at each time, according to their channel conditions. Assume each link has unit bandwidth. The matrix version of the optimization problem is:

$$
\min _{\gamma_{i}} \sum_{i=1}^{K} P_{i}
$$

$$
\text { subject to }\left\{\begin{array}{l}
\text { Feasible: }(I-D F) \mathbf{P} \geq \mathbf{u} \\
\text { Network Performance: } \sum_{i=1}^{K} T_{i}(n)=\mathrm{R} \\
\text { Fairness: } \lim _{N \rightarrow \infty} \sum_{n=1}^{N} T_{i}(n) / N=\text { const. }
\end{array}\right.
$$

where $T_{i}(n)=\log _{2}\left(M_{i}(n)\right)$, which is the $i^{t h}$ user's throughput, $\mathrm{R}$ is a constant. $\mathbf{P}=\left[P_{1}, \ldots, P_{K}\right]^{T}$, $\mathbf{u}=\left[u_{1}, \ldots, u_{K}\right]^{T}, \quad u_{i}=\gamma_{i} \mathbf{w}_{i}^{H} N_{i} \mathbf{w}_{i} / \rho_{i i} G_{i i}, \quad D=$ $\operatorname{diag}\left\{\gamma_{1}, \ldots, \gamma_{K}\right\}$ and

$$
\left[F_{i j}\right]= \begin{cases}0 & \text { if } j=i ; \\ \frac{\rho_{j i} G_{j i}\left\|\mathbf{w}_{i}^{H} \mathbf{h}_{j i}\right\|^{2}}{\rho_{i i} G_{i i}} & \text { if } j \neq i\end{cases}
$$

In order to solve the problem above, we divide the our algorithm in two steps. The first step is to ensure that $\lim _{N \rightarrow \infty} \sum_{n=1}^{N} T_{i}(n) / N=$ const.. In this step, the throughput range at different time that a user can select is determined. Then in the second step, we decide how to manage the powers and modulation levels within each user's throughput ranges at each time.

\section{B. Each User's Throughput Management}

Instead of having fixed throughput range $\left[T_{\min }, T_{\max }\right]$ for each link, we can adaptively change the throughput range, which takes into account of the links' throughput history. Suppose the $i^{\text {th }}$ link can select throughput in $T_{i}^{\text {min }}(n) \leq T_{i}(n) \leq T_{i}^{\max }(n)$ at time $n$ and the targeted 
the minimum and maximum throughput respectively that the $i^{\text {th }}$ link can select. The values are fixed and predefined by the system. In order to track the history of $T_{i}$, we define $T_{i}^{\text {mid }}(n)=T_{i}^{\text {mid }}(n-1)+\left(T_{i}(n)-T_{i}^{\text {ave }}\right) * \beta, \quad 0<\beta<1$, where $\beta$ is a constant that is depended on how fast the channel changes. If the channel changes fast, $\beta$ should select a relatively larger number, so that the SINR range can keep track of the channel changes. If the channel changes slowly, $\beta$ should select a relatively smaller number to have smooth effect. The value can be determined by some dynamic programming method. Here for simplicity, we assume $\beta$ is a constant. In each iteration, $T_{i}^{\min }(n), T_{i}^{\max }(n)$ and $T_{i}^{\text {mid }}(n)$ are updated by the algorithm in Table I.

TABLE I

Adaptive Algorithm for Throughput Range

\begin{tabular}{|l|}
\hline Initial: \\
\hline$T_{i}^{\text {min }}(0)=\hat{T}_{i}^{\text {min }}, T_{i}^{\text {max }}(0)=\hat{T}_{i}^{\text {max }}, T_{i}^{\text {mid }}(0)=T_{i}^{\text {ave }}$ \\
\hline Iteration: \\
\hline$T_{i}^{\text {mid }}(n)=T_{i}^{\text {mid }}(n-1)+\beta\left(T_{i}(n)-T_{i}^{\text {ave }}\right) ;$ \\
$T_{i}^{\text {min }}(n+1)=$ \\
$\min \left(\max \left(T_{i}^{\text {ave }}-T_{i}^{\text {mid }}(n)+\hat{T}_{i}^{\text {min }}, \hat{T}_{i}^{\text {min }}\right), \hat{T}_{i}^{\text {max }}\right) ;$ \\
$T_{i}^{\text {max }}(n+1)=$ \\
$\max \left(\min \left(\hat{T}_{i}^{\text {max }}-T_{i}^{\text {mid }}(n)+T_{i}^{\text {ave }}, \hat{T}_{i}^{\text {max }}\right), \hat{T}_{i}^{\text {min }}\right)$ \\
\hline
\end{tabular}

When $T_{i}(n)$ is continuously less than $T_{i}^{a v e}$ for some time, the $T_{i}^{\min }(n)$ is increased to $T_{i}^{a v e}$. Then the next $T_{i}(n+1)$ have to select throughput equal to or greater than $T_{i}^{a v e}$, consequently $T_{i}^{\text {mid }}(n)$ stops increasing. The same analysis can be applied to $T_{i}^{\max }(n)$. Since $T_{i}^{\min }(n)$ and $T_{i}^{\max }(n)$ are bounded and they are linearly modified by $T_{i}^{\text {mid }}(n)$, the $T_{i}^{\text {mid }}(n)$ is also bounded. Rearrange the $T_{i}^{\text {mid }}(n)$ and sum with the different time. We have

$$
\frac{\sum_{n=1}^{N} T_{i}(n)}{N}=T_{i}^{a v e}+\frac{\left(T_{i}^{\text {mid }}(N)-T_{i}^{a v e}\right)}{\beta N}
$$

Since $T_{i}^{\text {mid }}(N)$ is bounded, the second term in the right hand side decreases to zero as $N \rightarrow \infty$. So we prove that $\lim _{N \rightarrow \infty} \frac{\sum_{n=1}^{N} T_{i}(n)}{N}=T_{i}^{a v e}$.

\section{Optimal Throughput Allocation for Different Users}

In this subsection, we will develop adaptive algorithms that find the optimal throughput allocation for different users at each time. Because there are only limited numbers of $M_{i}$ that each link can select, we can use full search to find the optimal modulation allocation. The constraint is to keep the network throughput as a constant R. Under this constraint, the overall transmitted powers required by all combinations of $M_{i}$ are calculated. The modulation allocation that generates the lowest overall transmitted power is selected. The optimal adaptive algorithm is given in Tab.II:

The adaptive algorithm A has high complexity. When the number of users grows, the complexity is increased exponentially, which is not acceptable in practice. We need
TABLE II

Adaptive Algorithm A

\begin{tabular}{|l|}
\hline Initial: \\
\hline$T_{1}(0)=T_{1}^{\text {ave }} \ldots T_{K}(0)=T_{K}^{\text {ave }}$ \\
$P_{1}(0), \ldots, P_{K}(0)=$ any positive feasible const. \\
\hline Adaptive Throughput Allocation: \\
\hline search all possible $T_{i}(n)$ for every link \\
subject to $\sum_{1}^{K} T_{i}(n)=R$. \\
find the combination of $T_{i}(n)$ that \\
minimize $P_{\text {sum }}=\sum_{1}^{K} P_{i}\left(T_{i}(n)\right)$ \\
calculated by the iteration \\
\hline Iteration: \\
\hline Beamforming: $\mathrm{w}_{i}=\arg \max _{\mathrm{W}} \Gamma_{i}$ \\
\hline Power Allocation Update: \\
\hline$\gamma_{i}=S I N R$ for $T_{i}(n)$ and desired $B E R$ \\
$\quad \mathrm{P}=D F \mathrm{P}+\mathrm{u}$. \\
\hline Throughput Range Update: \\
\hline Update $T_{i}^{\text {mid }}(n), T_{i}^{\text {min }}(n), T_{i}^{\text {max }}(n)$. \\
\hline
\end{tabular}

to find fast algorithm with low complexity. First, in [5], the author find the gradients of overall transmitted power to adjust each user's targeted SINR to reduce the overall transmitted power. We need to find out which users contribute more to the overall transmitted power. If these users can sacrifice their targeted SINR (throughput) a little bit, the overall power is reduced significantly. We have the $i^{\text {th }}$ element of gradient $\mathbf{g}=\left[g_{1} \ldots g_{K}\right]^{T}$ of the overall uplink transmitting power $P_{\text {sum }}$ as:

$$
\begin{aligned}
g_{i} & =\frac{c_{i}\left(\mathbf{w}_{i}^{H} N_{i} \mathbf{w}_{i}+\sum_{j \neq i} P_{j} \rho_{j i} G_{j i}\left\|\mathbf{w}_{i}^{H} \mathbf{h}_{j i}\right\|^{2}\right)}{\rho_{i i} G_{i i}\left\|\mathbf{w}_{i}^{H} \mathbf{h}_{i i}\right\|^{2}} \\
& =\frac{c_{i} P_{i}}{S I N R_{i}}
\end{aligned}
$$

where $S I N R_{i}$ is the SINR detected at the base station's antenna diversity output for the $i^{t h}$ user, $c_{i}=\mathbf{1}^{T}(I-$ $D F)^{-1} \mathbf{v}_{i}$, and

$$
\left[\hat{\mathbf{v}}_{i}\right]_{j}=\left\{\begin{array}{l}
1, \text { if } j=i \\
0, \text { otherwise }
\end{array} .\right.
$$

The value of $c_{i}$ reflects how severe the co-channel interferences are. When the co-channel interferences are large, $c_{i}$ tells which user causes more co-channel interferences to other users. When the co-channel interferences are small, $c_{i} \approx c_{j} \forall i, j$. Since we only care the direction of the gradient and do not care the amplitude, we can ignore the value of $c_{i}$ when the co-channel interferences are small. Equ. 6 is very significant in that it provides very simple way to find the gradient. In this case, we can measure SINR at each base station's antenna diversity output and use the feedback channel to get the mobile transmitted power value to calculate the direction of gradient. Consequently the system complexity is reduced greatly. By using the gradient, we know how to optimally reduce the overall transmitted power. 
Now we introduce a greedy algorithm. We compare the gradients of different users. If the user with larger gradient selects lower code rate, i.e. it requires lower targeted SINR threshold, the overall transmitted power is reduced greatly. So first we find which code rate for the user with the highest gradient generates the lowest overall power subject to $T=R$. When we change the rate of the user with the highest gradient, we modify the rate of the user in the order from the smaller gradient to larger gradient to ensure the constraint. Then we find the optimal code rate for the user with the second highest gradient, and so on until the we find the rate of last user in the row. Because every user only searches fixed amount of coding rate and only reordering is needed, this sub-optimal algorithm has the complexity of only $O\left(N^{2} \log N\right)$. The algorithm is suboptimal because the optimal code rate or SINR threshold for one user may not be optimal for all the users.

Rearrange the user index from the largest gradient to the lowest. i.e. $g_{1} \geq g_{2} \geq \ldots \geq g_{K}$. The sub-optimal adaptive iterative algorithm at the $n^{\text {th }}$ iteration to find the modulation allocation with joint power control and beamforming is given in Tab. III.

TABLE III

Adaptive Algorithm B

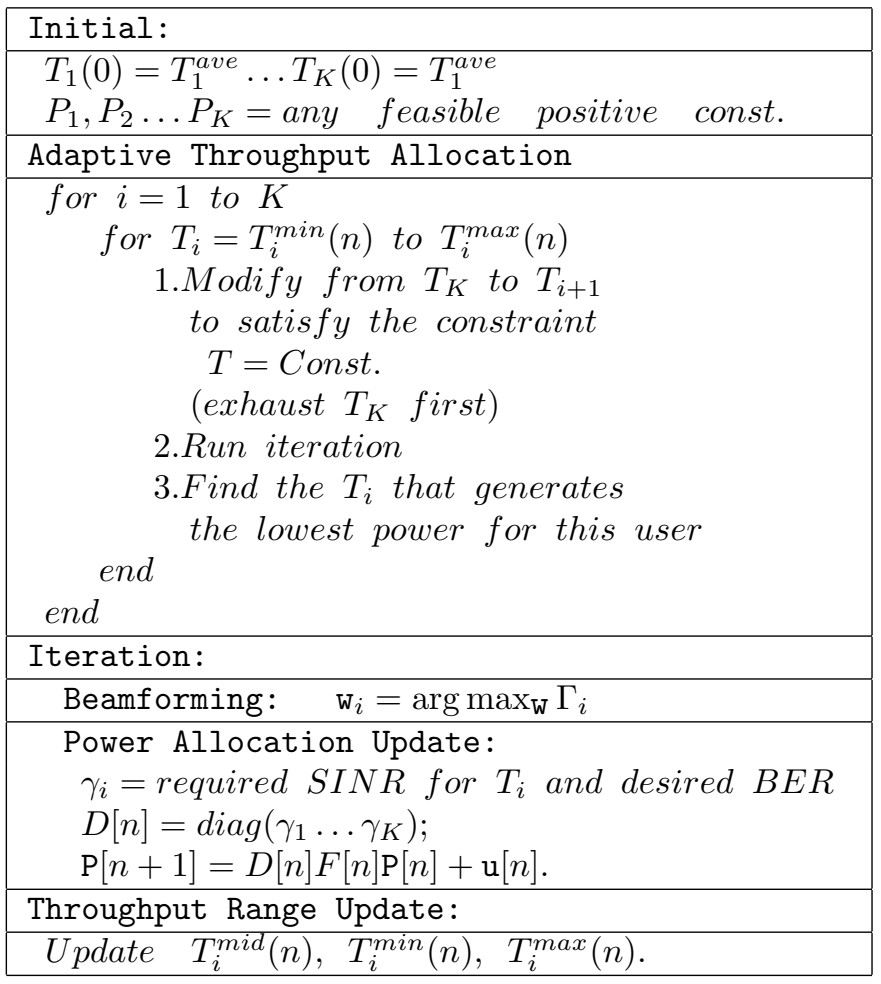

With the adaptive algorithm, we can construct a variable power and variable rate system as shown in Fig. 2 . The adaptive algorithm module gets estimation of users' channel responses from the channel estimation module. Then power control and modulation information are computed. The power control information and best modulation method are sent back to mobiles.

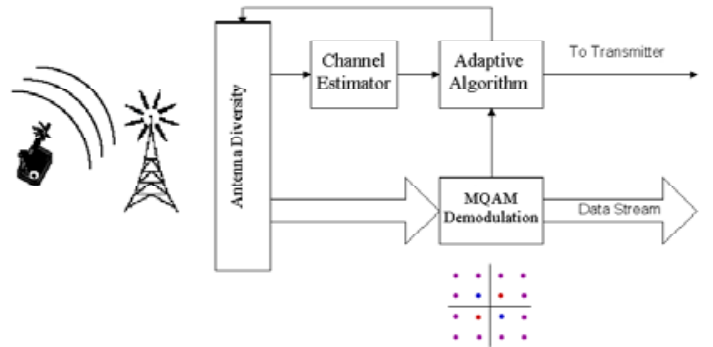

Fig. 2. Variable Power and Variable Rate System

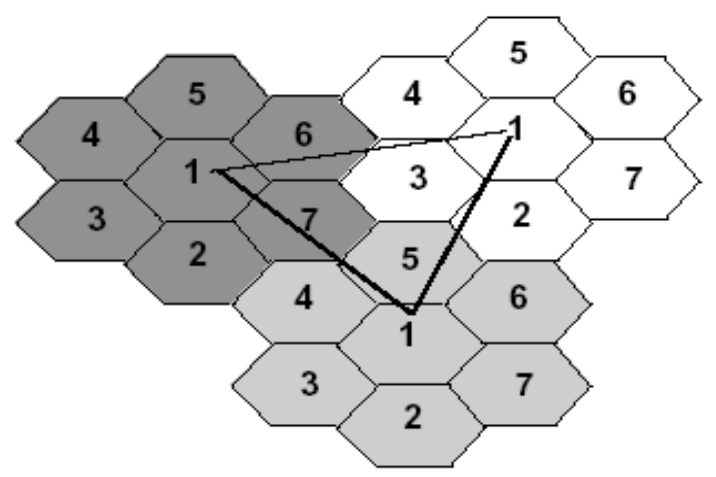

Fig. 3. Simulation Setup

\section{Simulation Results}

In order to evaluate the performance of our algorithms, a network with hexagonal cells is simulated as shown in Fig. 3. The radius of each cell is $1000 \mathrm{~m}$. The number in each cell represents the assigned channel number. Two adjacent cells do not share the same channel. There are 50 cells in the networks. One base station is placed at the center of the each cell. In each cell, one user is placed randomly with a uniform distribution. In the simulation, we consider three multipaths with equal power Rayleigh fading with equal power. The delay spread between the different paths is far less than one symbol duration. Each base station has a four element of antenna array.

Path loss is due to the decay of the intensity of a propagating radio wave. In this paper, we use the two slope path loss model [6] to obtain the average received power as a function of distance. The average path loss is given by:

$$
G=\frac{C}{r^{a}\left(1+r \lambda_{c} /\left(4 h_{b} h_{m}\right)\right)^{b}}
$$

where $C$ is a constant, $r$ is the distance between the mobile and the base station, $a$ is the basic path loss exponent (approximately two), $b$ is the additional path loss component (range from two to six), $h_{b}$ is the base station antenna height, $h_{m}$ is the mobile antenna height, and $\lambda_{c}$ is the wavelength of the carrier frequency. We assume the mobile antenna height is $2 \mathrm{~m}$ and the base station antenna height is $50 \mathrm{~m}$. The carrier frequency is $900-\mathrm{MHz}$.

In the urban microcell system, the link quality is also 


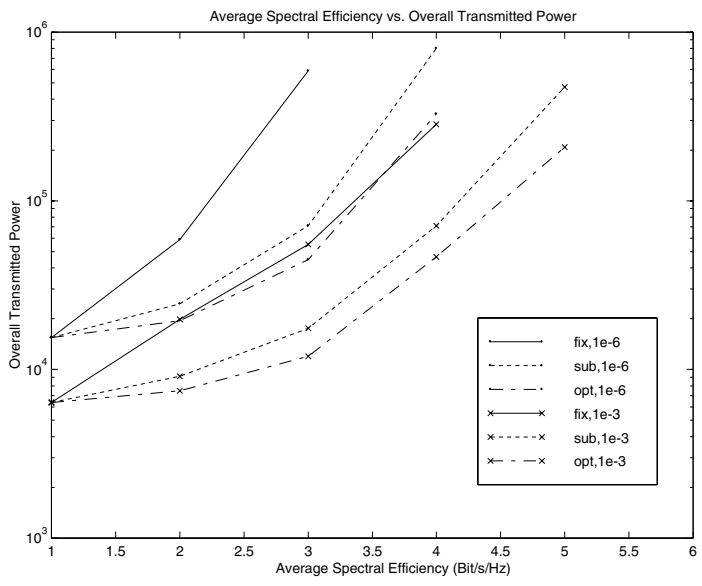

Fig. 4. Overall Power vs. Spectral Efficiency

affected by the shadowing of the line of sight path from terrain, buildings and trees. The shadowing is generally modeled as lognormal distribution. The probability density function $(\mathrm{PDF})$ is given by:

$$
P D F(\rho)=\frac{1}{\sqrt{2 \pi} \sigma \rho} \exp \left\{-\frac{(\log \rho-\xi)^{2}}{2 \sigma^{2}}\right\}, \rho>0
$$

where $\xi$ is related to path loss, $\sigma$ is shadow standard deviation. For each link, $3 \mathrm{~dB}$ deviation is considered.

In Fig.4, we compare the overall transmitted power as function of average spectral efficiency for fixed scheme (every links use the same MQAM), suboptimal scheme and optimal scheme of $B E R=10^{-3}$ and $B E R=10^{-6}$ at a snapshot. From the curves we can see that our algorithm greatly reduces the overall transmitted power and increase the maximum achievable throughput. The suboptimal algorithm has the performance between the fixed scheme and optimal scheme.

In Fig.5 and Fig.6, we show the average power saving and average spectral efficiency gain as the function of window size $\left(\max \left(\hat{T}_{i}^{\max }-T_{i}^{\text {ave }}, T_{i}^{\text {ave }}-\hat{T}_{i}^{\text {min }}\right)\right)$ for a time period of 1000. The power stops decreasing and spectral efficiency stops increasing as the window size growing. This is because of the time average throughput constraint for each link. The link who gets better throughput at this time must pay back in the future. The results show that our scheme can reduce up to $80 \%$ overall transmitted power when $B E R=10^{-3}, 66 \%$ overall transmitted power when $B E R=10^{-6}$ and increase spectral efficiency about 1.2 $\mathrm{bit} / \mathrm{s} / \mathrm{Hz}$.

\section{Conclusion}

On the whole, by adaptively managing links' power and modulation, we can greatly improve network performance. Our scheme can be interpreted as "water filling" each link's throughput in time domain and allocating the network throughput to different links at each time. We propose two sets of adaptive algorithms, which gives valuable in-

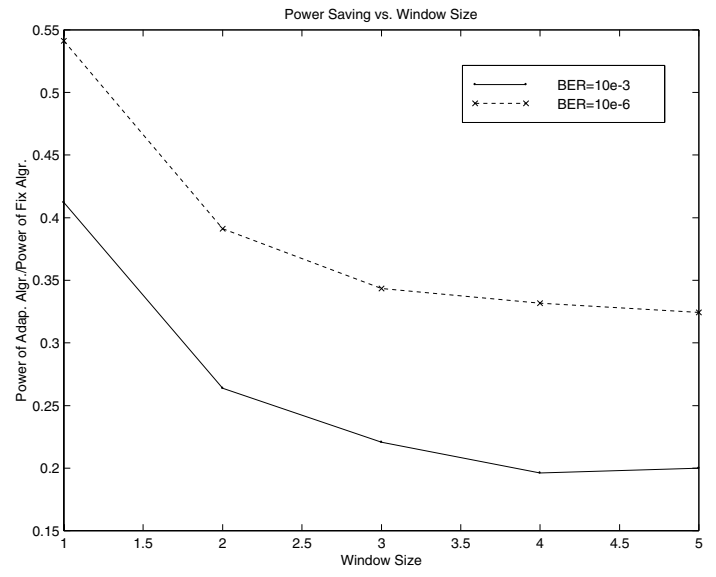

Fig. 5. Power Saving vs. Window Size

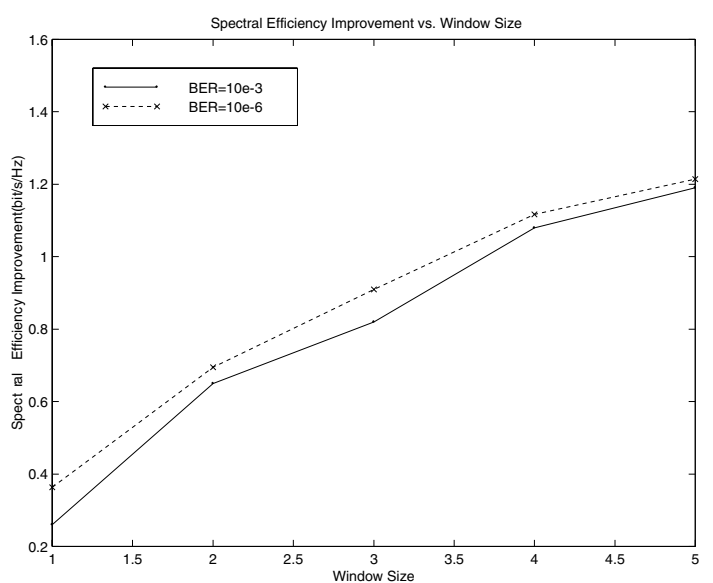

Fig. 6. Spectral Efficiency Gain vs. Window Size

sight in the adaptive resource allocation in multi-user environments.

From our simulation results, the algorithm reduces up to $80 \%$ of the total transmitting power of mobile users, which is very critical in terms of battery life. The spectral efficiency is increased about $1.2 \mathrm{bit} / \mathrm{s} / \mathrm{Hz}$, which in turn increases the whole network throughput.

\section{REFERENCES}

[1] F.Rashid-Farrokhi, L. Tassiulas, K.J. R. Liu, "Joint optimal power control and beamforming in wireless network using antenna arrays", IEEE Trans. Commun., vol.46, no.10, pp.1313-1323, Oct. 1998 .

[2] R. Yates, "A framework for uplink power control in cellular radio systems", IEEE J. Sel. Areas Commun., vol.13, no.7, pp.13411348, Sep. 1995.

[3] X. Qiu and K. Chawla, "On the performance of adaptive modulation in cellular systems," IEEE Trans. Commun., vol.47, no.6, pp.884-895, Jun. 1999.

[4] S.T. Chung and A.J. Goldsmith "Degrees of freedom in adaptive modulation: a unified view", IEEE Trans. Commun. , vol.49, pp.1561-1571, Sep. 2001.

[5] Z. Han, K.J.R. Liu, "Adaptive SINR threshold allocation for joint power control and beamforming over wireless network", IEEE VTC., pp.1548-1552, Fall 2001.

[6] P. Harley, "Short distance attenuation measurements at $900 \mathrm{MHz}$ and $1.8 \mathrm{GHz}$ using low antenna heights for microcells", IEEE Trans. Sel. Areas Commun., vol.37, pp.220-222, Nov. 1988. 